\title{
Article \\ Loss of the Extracellular Matrix Protein DIG-1 Causes Glial Fragmentation, Dendrite Breakage, and Dendrite Extension Defects
}

\author{
Megan K. Chong ${ }^{1,2,+}$, Elizabeth R. Cebul ${ }^{1,2,+} \mathbb{D}$, Karolina Mizeracka ${ }^{1,2,+}$ and Maxwell G. Heiman ${ }^{1,2, *(D)}$ \\ 1 Department of Genetics, Harvard Medical School, Boston, MA 02115, USA; \\ chongmegank@gmail.com (M.K.C.); elizabeth.r.cebul@gmail.com (E.R.C.); kmizeracka@gmail.com (K.M.) \\ 2 Division of Genetics and Genomics, Boston Children's Hospital, Boston, MA 02115, USA \\ * Correspondence: heiman@genetics.med.harvard.edu \\ + These authors contributed equally to this work.
}

Citation: Chong, M.K.; Cebul, E.R.; Mizeracka, K.; Heiman, M.G. Loss of the Extracellular Matrix Protein DIG-1 Causes Glial Fragmentation, Dendrite Breakage, and Dendrite Extension Defects. J. Dev. Biol. 2021, 9, 42. https://doi.org/10.3390/jdb9040042

Academic Editors: Brian Ackley and Simon J. Conway

Received: 12 August 2021

Accepted: 24 September 2021

Published: 7 October 2021

Publisher's Note: MDPI stays neutral with regard to jurisdictional claims in published maps and institutional affiliations.

Copyright: (c) 2021 by the authors. Licensee MDPI, Basel, Switzerland. This article is an open access article distributed under the terms and conditions of the Creative Commons Attribution (CC BY) license (https:/ / creativecommons.org/licenses/by/ $4.0 /)$.

\begin{abstract}
The extracellular matrix (ECM) guides and constrains the shape of the nervous system. In C. elegans, DIG-1 is a giant ECM component that is required for fasciculation of sensory dendrites during development and for maintenance of axon positions throughout life. We identified four novel alleles of dig-1 in three independent screens for mutants affecting disparate aspects of neuronal and glial morphogenesis. First, we find that disruption of DIG-1 causes fragmentation of the amphid sheath glial cell in larvae and young adults. Second, it causes severing of the BAG sensory dendrite from its terminus at the nose tip, apparently due to breakage of the dendrite as animals reach adulthood. Third, it causes embryonic defects in dendrite fasciculation in inner labial (IL2) sensory neurons, as previously reported, as well as rare defects in IL2 dendrite extension that are enhanced by loss of the apical ECM component DYF-7, suggesting that apical and basolateral ECM contribute separately to dendrite extension. Our results highlight novel roles for DIG-1 in maintaining the cellular integrity of neurons and glia, possibly by creating a barrier between structures in the nervous system.
\end{abstract}

Keywords: neurodevelopment; dendrites; glia; extracellular matrix; DIG-1; C. elegans

\section{Introduction}

Neurons and glia exhibit a kaleidoscopic array of shapes, with each cell type adopting a distinctive morphology that is intimately coordinated with its immediate neighbors [1]. Most studies of cell morphogenesis have focused on factors that sculpt cells from the inside, especially regulators of the cytoskeleton. By contrast, unbiased genetic approaches have pointed to an equally important role for factors that act from outside the cell-in particular, components of the extracellular matrix (ECM).

C. elegans provides a powerful system to identify novel factors that shape neurons and glia. Briefly, the morphology of every neuron and glial cell is highly stereotyped and has been carefully catalogued; individual cells can be readily visualized in live intact animals throughout life; and it is straightforward to perform forward genetic screens by visually identifying mutants that show aberrant morphology of single neurons or glial cells. Recent screens have revealed roles for extracellular factors (i.e., ECM components and cell surface adhesion molecules) in diverse aspects of neurodevelopment, including neuroblast migration [2-4], axon and dendrite extension [5-9], fasciculation of neurites into nerve bundles [10-15], and synapse development [16-21].

By examining C. elegans sense organs, we previously identified several extracellular factors that control neuronal and glial morphogenesis [9,10,22-26]. We initially focused on the major sense organ, the amphid, which consists of 12 sensory neurons and two glial cells, called the amphid sheath—which is examined further in this study-and the amphid 
socket [27]. The neurons extend unbranched sensory dendrites to the nose tip, where most of them protrude into the external environment through a pore formed by the sheath and socket glia [27]. We showed that this structure develops embryonically as a narrow epithelial tube lined by the apical ECM protein DYF-7 [9]. In the absence of DYF-7, this tube ruptures, causing the sensory neurons and sheath glial cell to detach from the nose and resulting in severely shortened dendrites [9,22].

In other sense organs, sensory dendrites protrude through pores in their own dedicated glia. For example, the IL2 neurons-another cell type examined in this studyprotrude through pores in IL sheath and socket glia. We previously found that these neurons also require DYF-7 for dendrite extension, suggesting that they similarly develop as part of an epithelial tube [9]. By contrast, some sensory dendrites extend to the nose tip but do not pass through a glial pore and do not require DYF-7. For example, the carbon dioxide-sensing neuron BAG - the third cell type examined in this study-forms elaborate membranous attachments along the surface of a specific glial partner but does not enter a glial pore [9]. Unlike the amphid and IL2 neurons, BAG does not require DYF-7 for dendrite morphogenesis, but instead requires a cell surface adhesion molecule that acts both in the neuron itself and in glia [23]. Together, these examples illustrate how neurons and glia can be shaped by extracellular factors that mediate specific physical interactions.

Here, we report that three genetic screens for mutants affecting disparate cellular phenotypes-amphid sheath glial cell morphology, IL2 dendrite extension, and BAG dendrite integrity-all identified lesions in the same gene, encoding the giant ECM molecule DIG-1. DIG-1 was previously shown to affect neuronal cell body positioning and the fasciculation of axons and dendrites, including amphid and IL2 dendrites [11,12,28,29]. We find that loss of DIG-1 also causes a novel glial fragmentation defect, breakage of BAG dendrites, and detachment of IL2 dendrites from the nose tip. Glial fragmentation and BAG dendrite breakage occur in late larval and early adult stages, while IL2 dendrite defects appear to arise during embryonic development and are enhanced by loss of DYF-7, which also acts embryonically. These observations raise the question of how a single ECM component affects such diverse neuronal and glial cell types with distinctive outcomes and different developmental timing. We consider the possibility that DIG-1 protects the structural integrity of neurons and glia by creating a barrier between them and their surroundings.

\section{Materials and Methods}

\subsection{Strains and Strain Maintenance}

All strains were constructed in the $\mathrm{N} 2$ background and cultured at $20^{\circ} \mathrm{C}$ on nematode growth medium (NGM) plates seeded with E. coli OP50. Strains, transgenes, and alleles used in this study are listed in Supplementary Tables S1-S3.

\subsection{Forward Genetic Screens and Genetic Mapping}

All mutants were isolated from F2 nonclonal forward genetic screens. Briefly, L4 animals carrying an integrated cell-specific reporter (amphid sheath: F16F9.3pro:mCherry (CHB1549 hmnIs13); BAG: flp-17pro:GFP (PY8503 oyIs82); IL2: klp-6pro:GFP (PT2660 myIs13)) were mutagenized for $4 \mathrm{~h}$ at room temperature in $70 \mathrm{mM}$ ethyl methanesulfonate (EMS, Sigma, Saint Louis, MO, USA). Nonclonal F2 progeny were visually inspected for altered cellular morphologies using a Nikon SMZ1500 stereomicroscope with an HR Plan Apo 1.6× objective. Animals with aberrant cell shapes were recovered to individual plates. hmn152, $h m n 158, h m n 227$, and $h m n 259$ mutations were crossed to the Hawaiian wild strain CB4856 and mapped to the dig- 1 locus by whole-genome sequencing of pooled recombinants [30,31]. The sequence mutations identified in each allele are listed in Supplementary Table S3.

\subsection{Recapitulation and Complementation Tests Using the $n 1321$ Reference Allele}

After identifying dig-1 lesions in each mutant strain, the reference allele dig-1(n1321) was examined. Briefly, the strain MT2840 (dig-1(n1321)) was obtained from the C. elegans Genetics Center (CGC) and crossed to markers for the amphid sheath (hmnIs13), BAG 
neuron (oyIs82), and IL2 neurons (myIs14 [klp-6pro:GFP] was used instead of myIs13 because the latter is on the same chromosome as dig-1). For complementation tests, MT2840 (n1321) males were crossed to each of the four strains shown in Supplementary Figure S1 (CHB1652 (hmn227; hmnIs13), CHB1166 (hmn152; oyIs82), CHB2250 (hmn158; oyIs82), and CHB3008 (hmn259; myIs13)) and heterozygous cross-progeny were examined at the L4 stage. The following defects were observed: MT2840 $\times$ CHB1652, amphid sheath defects in 10/22 animals; MT2840 $\times$ CHB1166, BAG defects in 5/27 animals; MT2840 $\times$ CHB2250, BAG defects in 9/24 animals; MT2840 $\times$ CHB3008, IL2 defects in 8/17 animals.

\subsection{Microscopy and Image Processing}

Animals were immobilized with 10-100 mM sodium azide dissolved in M9, depending on developmental stage, mounted on a $2 \%$ agarose pad, and covered with a No. 1.5 coverslip. Spherical aberration was minimized using immersion oil matching. Z-stacks were acquired using a DeltaVision Core deconvolution imaging system (Applied Precision) with the InsightSSI light source; UApo $40 \times / 1.35$ NA oil immersion objective, PlanApo $60 \times / 1.42$ NA oil immersion objective, or UPlanSApo $100 \times / 1.40$ NA oil immersion objective (Olympus); the standard DeltaVision live cell excitation and emission filter set; and a Photometrics CoolSnap HQ2 CCD camera (Roper Scientific). Images were acquired and deconvolved with Softworx 5.5 (Applied Precision). Images are displayed as maximum intensity projections generated in Priism [32]. Images were pseudocolored and image brightness was linearly adjusted using Adobe Photoshop. IL2 dendrite lengths were measured using the Segmented Line tool in Fiji (NIH) [33]. To control for differences in head size, dendrite lengths are normalized to the distance from the cell body to the nose tip. $p$-values were generated using the Wilcoxon Rank-Sum (Mann-Whitney U) test in RStudio and adjusted using the Bonferroni correction.

\section{Results}

\subsection{Genetic Screens for Disparate Glial and Neuronal Phenotypes Identify Lesions in DIG-1}

To identify factors that control the morphology of glia and neurons, we performed visual forward genetic screens using cell-type-specific fluorescent markers. Briefly, we mutagenized strains bearing markers for the amphid sheath glial cell (F16F9.3pro:mCherry), the sensory neuron BAG (flp-17pro:GFP), or the sensory neuron IL2 (klp-6pro:GFP); allowed animals to self-fertilize for two generations to yield F2 progeny bearing random homozygous mutations; and visually screened nonclonal pools of progeny using a fluorescence stereomicroscope to isolate individuals with aberrant cell morphologies (Figure 1A). Four mutants (hmn152, hmn158,hmn227, and hmn259) in which the phenotype of interest was transmitted with high penetrance across generations were analyzed further (Figure 1A, Supplementary Figure S1). All four mutants were recessive, and we used whole-genome sequencing of pooled recombinants to identify possible causative mutations.

Lesions in dig- 1 were identified in mutants from each screen (Figure 1B). dig- 1 is predicted to encode a 13,084 amino acid ECM protein consisting of an amino-terminal region comprised of repetitive Immunoglobulin (Ig)-like and Fibronectin III (FN)-like domains that suggest roles in cell adhesion, a large central region of poorly conserved sequence with unknown function, and a carboxy-terminal region containing von Willebrand Factor A (VWA) and epidermal growth factor (EGF)-like domains that are often found in ECM and cell adhesion proteins (Figure 1B) [11,12]. It contains two RGD motifs, suggesting a possible role in integrin binding (Figure 1B). Three of the alleles we isolated are predicted to disrupt a splice site ( $h m n 158$ and $h m n 227$; these were isolated in independent screens but result in an identical nucleotide change), or introduce a premature termination codon (hmn152), suggesting they may represent severe loss of function mutations (Figure 1B). Interestingly, one allele ( $h m n$ 259) introduces a Pro > Ser missense mutation at position 3710 in a poorly conserved region, pointing to a possible functional role for this otherwise unannotated sequence (Figure 1B). Interestingly, a previously reported allele, nu336, introduces 
a Ser > Phe missense mutation in approximately the same region, at position 3988 [11]. It is worth noting that both alleles were isolated in screens for IL2 morphogenesis defects.

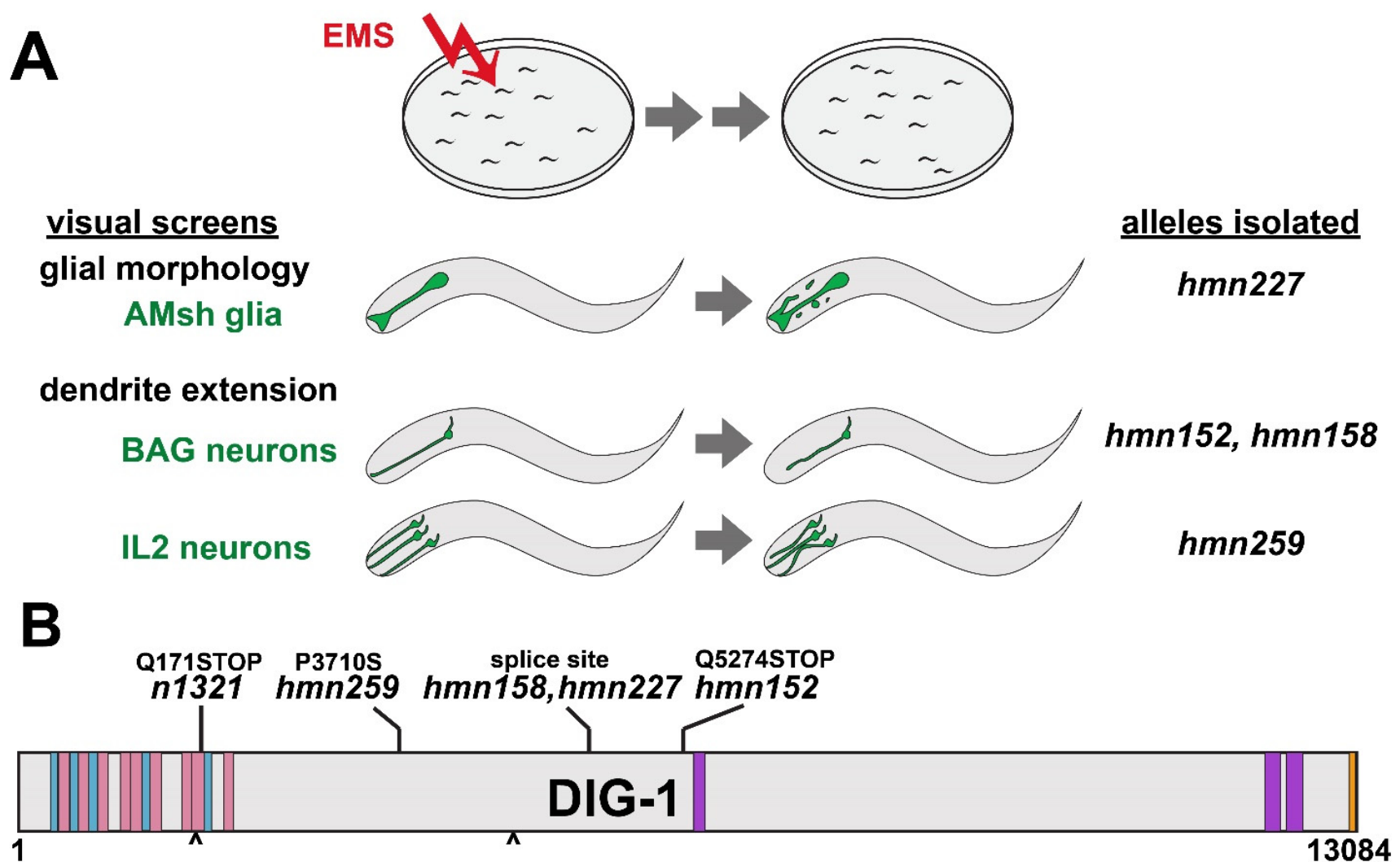

Figure 1. Overview of genetic screens and dig-1 alleles. (A) Schematic of screening strategy, phenotypes, and resulting alleles isolated. Animals bearing markers for the amphid sheath (AMsh) glia, BAG neurons, or IL2 neurons were mutagenized with ethyl methanesulfonate (EMS), grown for two generations, and screened for morphology defects, yielding four alleles of dig-1. (B) Schematic of DIG-1 protein depicting the nature of each newly isolated allele as well as the reference allele $n 1321$ used throughout this study. Blue, Ig domains; pink, FN domains; purple, VWA domains; orange, EGF domain; carets, RGD motifs.

Three lines of evidence argue that dig-1 lesions are causal for the phenotypes we identified. First, sequencing of pooled recombinants showed that the lesions are genetically linked to the phenotypes. Second, the phenotypes were recapitulated using the reference allele dig-1(n1321), which is predicted to introduce a premature termination codon at position $171[12,34]$. Third, the reference allele failed to complement each of the alleles for their respective phenotypes $(n 1321 / \mathrm{hmn} 227$, amphid sheath defects in $10 / 22 \mathrm{~L} 4$ animals; $n 1321 / h m n 152$ and $n 1321 / h m n 158$, BAG defects in 5/27 and 9/24 L4 animals, respectively; n1321/hmn259, IL2 defects in 8/17 L4 animals; see Materials and Methods). We did not perform transgenic rescue experiments due to the size of the dig- 1 gene and cDNA (48 kb and $39 \mathrm{~kb}$ respectively). However, taken together, the linkage, recapitulation, and noncomplementation results strongly suggest that the dig-1 lesions are causative for these phenotypes. For consistency, we used the reference allele dig-1(n1321) for all further experiments in this study.

\subsection{Loss of DIG-1 Causes Glial Fragmentation in Young Adults}

The amphid is the largest sense organ in C. elegans, consisting of 12 neurons and the sheath and socket glia [27]. The sheath develops in concert with the neurons, together forming a multicellular rosette in the embryo that re-organizes during embryo morphogenesis to form an epithelial tube in which the sheath glial cell wraps the dendrite endings $[9,25]$. 
In the mature structure, the amphid sheath cell body is located near those of the neurons and extends a process collateral with the amphid dendrites, terminating at the nose where it adopts a characteristic wing-shaped morphology [27]. The dendrites of the 12 amphid neurons penetrate into the sheath, with the sheath forming ring-shaped tight junctions around each dendrite individually $[9,27,35]$. Four neurons form elaborate ciliated endings that are embedded in the wing-shaped portion of the sheath, while the others terminate in simple cilia that lie in a central lumenal channel $[27,35]$. Previous genetic screens have identified mutants that affect amphid sheath specification and cell body positioning [36] that disrupt extension of the amphid sheath glial process $[9,22]$, or that lead to enlarged or supernumerary amphid sheath cells [37,38].

In dig- 1 mutant animals, we observed a defect that, to our knowledge, has not been seen in other mutants affecting amphid sheath development. Although the amphid sheath appears superficially normal in newly hatched first larval stage (L1) animals, it exhibits progressively more severe disorganization in L4 and two-day adult (2A) animals (Figure 2). The characteristic wing-shaped ending becomes enlarged, extending more posteriorly along the head (Figure 2B,C). Abnormal protrusions or empty spaces (vacuoles) are often seen at the cell ending (Figure 2B,C). Most strikingly, the glial cell appears to fragment, with a multitude of $\sim 1-5 \mu \mathrm{m}$ fluorescent blebs appearing around the sheath glial process (Figure 2B,C). The distribution of these fragments suggests they may be internalized by neighboring cells. Interestingly, the amphid sheath was recently shown to internalize extracellular vesicles released by amphid neurons and, in the absence of the sheath, other neighboring cells perform this function, suggesting that several cells in the head can internalize cellular debris [39]. This phenotype is highly penetrant, with $>80 \%$ of individual amphid sheath glial cells showing extensive fragmentation (Figure 2D). Although we did not directly visualize the pore formed by the amphid sheath and socket glia, amphid neurons stain normally with the lipophilic dye DiO in dig-1 animals [11], suggesting that the pore remains open.

\subsection{Loss of DIG-1 Causes Breakage of BAG Dendrites in Young Adults}

BAG is a carbon dioxide-sensing neuron that forms a specialized attachment to a specific glial partner, the inner labial socket (ILso) glial cell [23,27]. Like other head sensory neurons, BAG extends an unbranched dendrite to the nose tip where it terminates in a sensory cilium. However, the BAG dendrite does not penetrate a sheath glial cell. Instead, its cilium forms a membranous "bag" that precisely wraps a thumb-like protrusion of the ILso glial cell $[23,27]$. During development, the BAG dendrite extends by attaching near the presumptive nose tip and then stretching out during embryo elongation [23]. BAG dendrite extension requires the adhesion molecule SAX-7, which acts both in BAG and in glia, as well as the cytoskeletal organizer GRDN-1, which acts in glia [23]. In the absence of SAX-7 or GRDN-1, the BAG dendrite detaches from the nose during embryo elongation, resulting in severely shortened dendrites in the mature structure [23].

While screening for additional mutants that affect BAG dendrite extension, we isolated two alleles of $d i g-1$ that exhibited an unusual phenotype. Unlike sax-7 and $g r d n-1$ mutants, in which BAG dendrites appear shortened from L1 through adulthood, dig-1 mutants exhibited superficially wild-type BAG dendrites at the L1 stage. However, at later stages, these dendrites appeared to undergo constriction at a stereotyped region of the distal dendrite, and ultimately appeared to break, especially as animals reached adulthood (Figure 3). In most cases, a brightly fluorescent remnant was still visible at the nose tip, suggesting that the specialized attachment of BAG to the ILso glial cell remained intact (Figure 3B,C). The proximal dendrites often appeared wavy or curved, resembling a tightly stretched string that has been cut (Figure 3B,C). Overall, this phenotype was highly penetrant, with $72 \%$ of BAG dendrites appearing fully severed in 2A animals (Figure 3D). An additional $18 \%$ of BAG dendrites had a thin, dimly fluorescent connection-often visible only at longer exposure settings-between the main dendrite and the remnant at the nose (Figure 3B-D, "constricted"). We noted that wild-type BAG neurons appear to exhibit 
dimmer fluorescence in this region, suggesting they may become thinner here, possibly due to compressive force from surrounding structures (Figure $3 \mathrm{~A}, \mathrm{C}$ ). This raises the possibility that DIG-1 protects BAG dendrites from breakage at a naturally occurring pinch point.
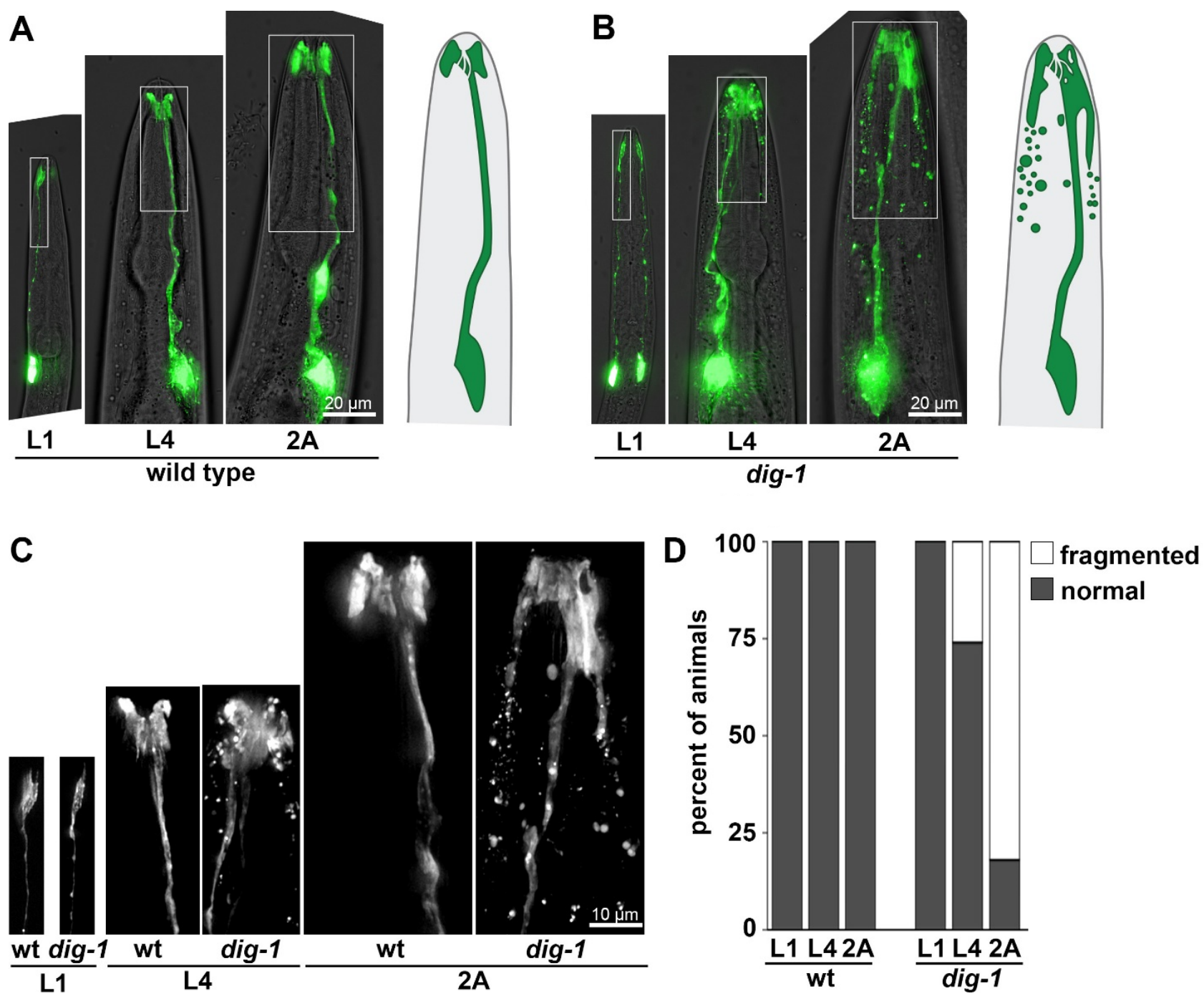

Figure 2. Amphid sheath glia undergo fragmentation in dig-1 mutants. Heads of (A) wild-type and (B) dig-1(n1321) animals at the L1, L4, and 2A stages, showing progressive fragmentation of amphid sheath in L4 and 2A dig-1 animals. Animals express F16F9.3pro:mCherry, pseudocolored green. Schematics of amphid sheath glia are shown on the right. (C) $2 \times$ magnification of boxed regions in (A,B). (D) Quantification of phenotype penetrance at each stage. $n=20$ animals per genotype at each stage. 

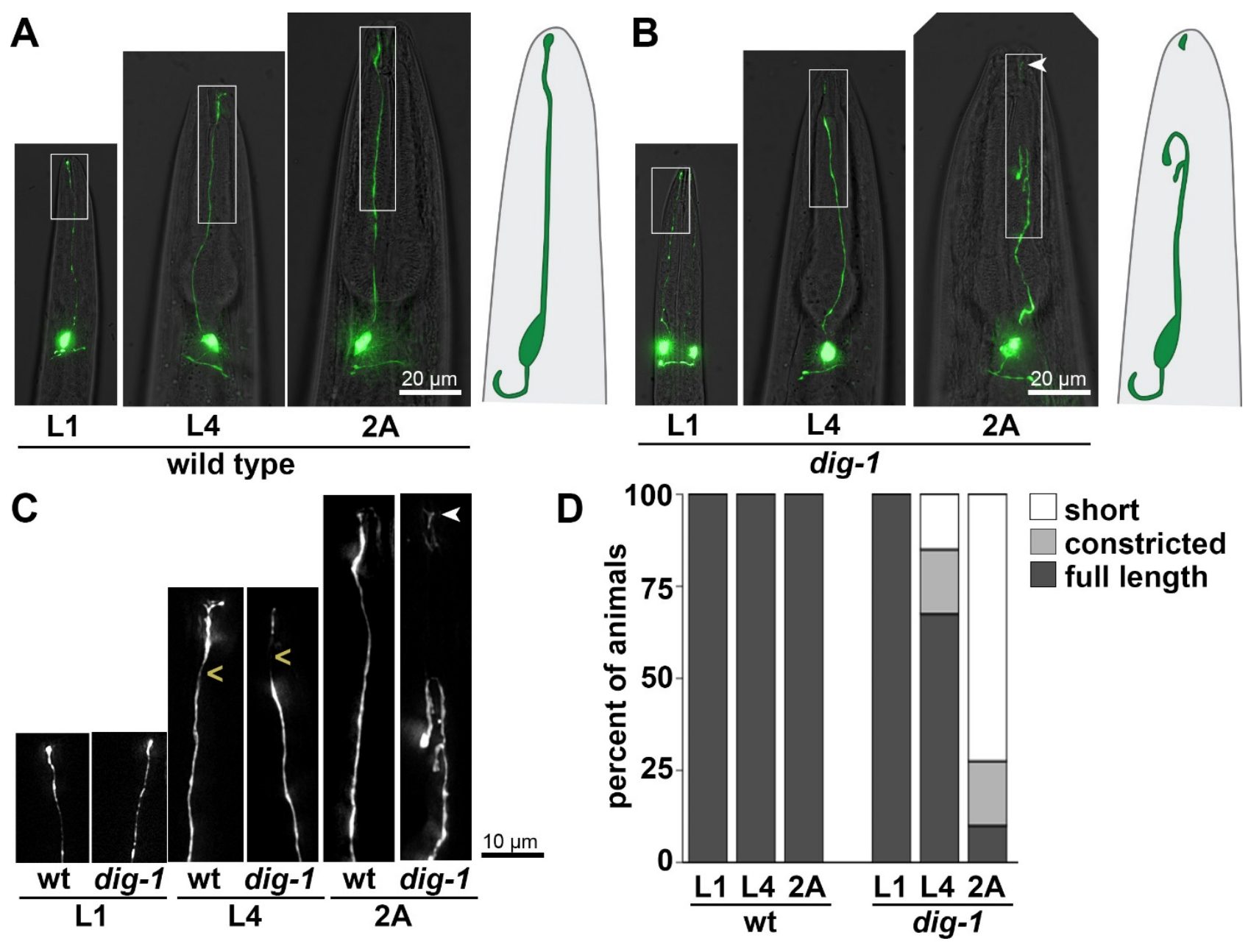

Figure 3. BAG dendrites constrict and break in dig-1 mutants. Heads of (A) wild-type and (B) dig-1(n1321) animals at the L1, L4, and 2A stages, showing apparent breakage of the BAG dendrite in L4 and 2A dig-1 animals. Animals express flp-17pro:GFP. Schematics of BAG neurons are shown on the right. (C) $2 \times$ magnification of boxed regions in (A,B). (D) Quantification of phenotype penetrance at each stage. "Constricted" refers to dendrites in which a thin, barely visible connection persists between the main dendrite and the remnant at the nose tip, as shown in the L4 dig-1 animal. This region (marked by $<$ ) appears thinner in wild-type animals as well. Arrowhead, remnant at the nose tip following dendrite breakage. $n=20$ animals per genotype at each stage.

\subsection{Loss of DIG-1 Causes Defasciculation and Dendrite Extension Defects in IL2 Neurons}

IL2 neurons are a set of six chemosensory neurons, arranged as dorsal, lateral, and ventral pairs [27]. Each IL2 dendrite penetrates its sheath glial cell as part of an epithelial tube, with its ciliated ending exposed directly to the outside environment. Similar to amphid neurons, IL2 neurons show a complete dependence on the apical ECM molecule DYF-7 for dendrite extension and, in $d y f-7$ mutants, $100 \%$ of IL2 neurons fail to reach the nose tip [9]. However, unlike amphid neurons, which become severely shortened in $d y f-7$ mutants ( $\sim-10 \%$ of wild-type length), IL2 neurons are more mildly affected ( $~ 80-90 \%$ of wild-type length) [9]. Therefore, to identify other factors that contribute to IL2 dendrite extension, we performed a visual screen for mutants with abnormal IL2 dendrites and identified dig-1 as a mutant with two abnormal aspects of IL2 dendrite morphology.

First, as has been described previously, loss of DIG-1 causes pronounced defasciculation defects in IL2 dendrites (Figure 4) [11]. Rather than extending directly to the nose tip in distinct parallel fascicles as in wild-type animals, IL2 dendrites took a meandering course in dig-1 mutants, often appearing to fasciculate together for some distance before separating again (Figure 4B,C). Other defasciculation defects have also been observed in dig-1 mutants, 
including in amphid dendrites [10,11] and several axon tracts [12], suggesting DIG-1 plays a general role in maintaining neurite bundles. However, while other defasciculation defects appear progressively throughout larval growth, consistent with previous work we find that IL2 defasciculation defects are present from the L1 stage with little change in phenotype penetrance as animals grow (Figure 4D) [11]. In addition to dendrite defects, IL2 neurons also exhibited variable cell body positioning at all stages, as reported previously [11]. It remains unclear why DIG- 1 is required embryonically for some nerve bundles, and only post-embryonically for others.
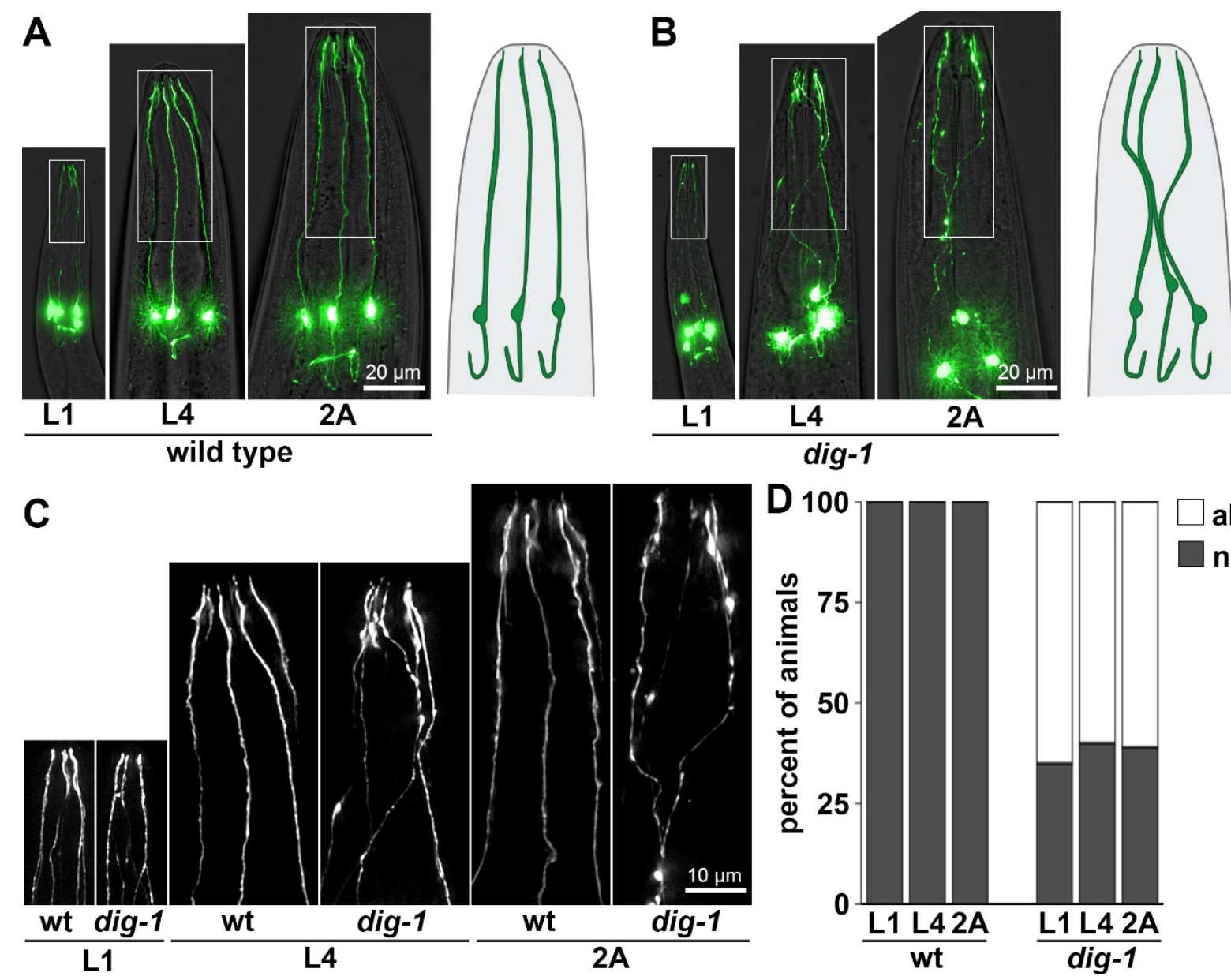

Figure 4. IL2 dendrites exhibit fasciculation defects in dig-1 mutants. Heads of (A) wild-type and (B) dig-1(n1321) animals at the L1, L4, and 2A stages, showing fasciculation defects at all stages in dig-1 animals. Animals express $k l p-6$ pro:GFP. Schematics of IL2 neurons are shown on the right. (C) $2 \times$ magnification of boxed regions in (A,B). (D) Quantification of phenotype penetrance at each stage. $n=20$ animals per genotype at each stage.

Second, we observed rare (6\%) dendrites that were less than half their normal length (Figure $5 \mathrm{C}, \mathrm{E}$ ), indicating a role for DIG-1 in dendrite extension. To test for a genetic interaction with $d y f-7$, we examined dig-1; $d y f-7$ double mutants using the presumptive null allele $d y f-7$ (ns119) which introduces a premature termination codon at position 3 [22]. We focused on L4 animals, because in L1 animals the smaller size and decreased brightness of dendrites combined with the fasciculation defects made it difficult to measure dendrite lengths accurately. In the dig-1; dyf-7 animals, the average IL2 dendrite length was shorter and dendrite lengths were overall more variable than in either single mutant (Figure 5D,E), suggesting that DIG-1 acts independently of DYF-7-that is, there is a role for DIG-1 in promoting IL2 dendrite extension even in the absence of DYF-7 (Figure 5). It is worth noting that DYF-7 is a component of the apical ECM, while DIG-1 is thought to primarily be 
present in basal ECM, suggesting that apical and basal ECM may contribute independently to sensory dendrite extension.

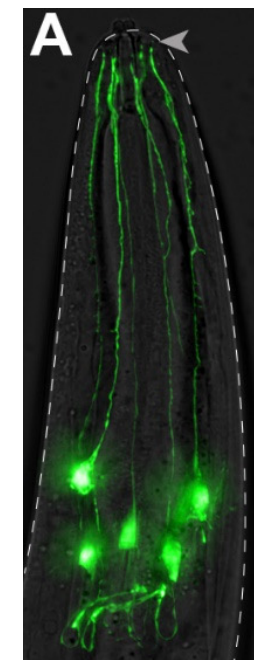

wild

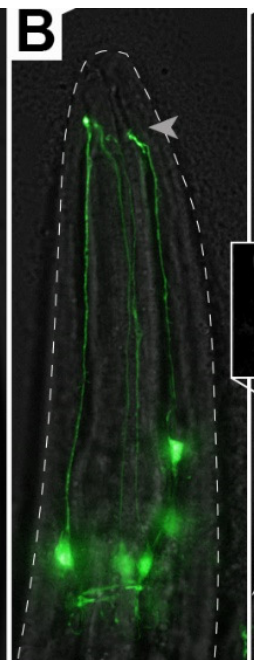

dyf-7

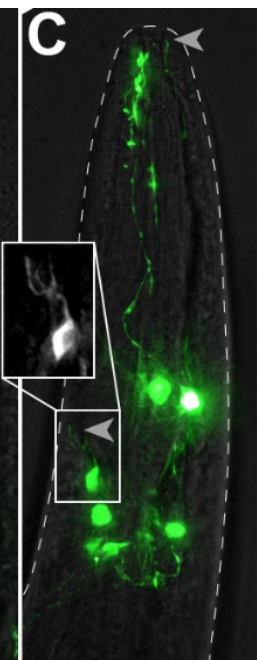

$\operatorname{dig}-1$

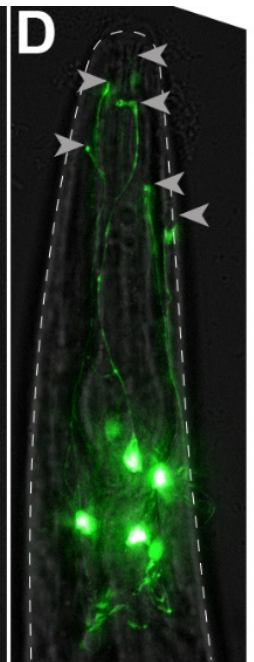

dig-1; dyf-7

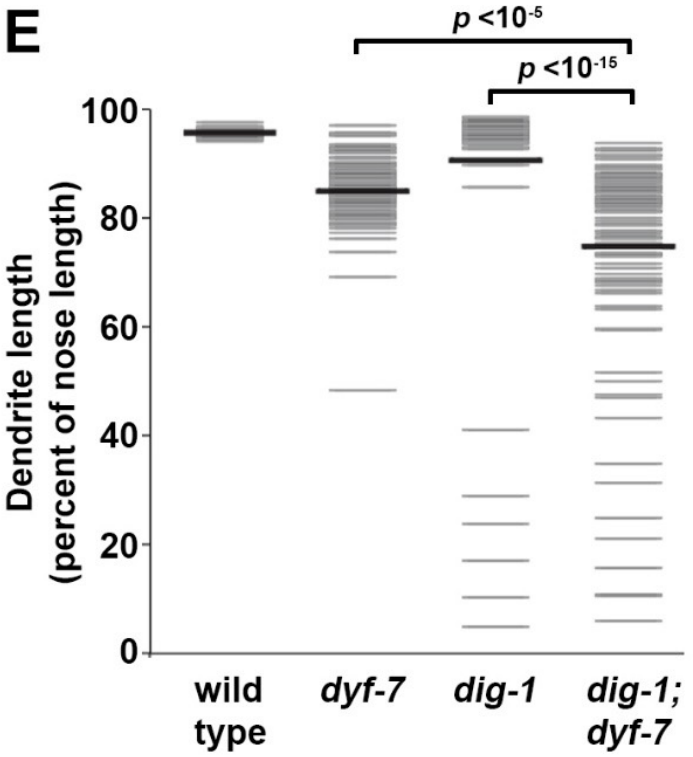

Figure 5. Rare IL2 dendrite extension defects in dig-1 mutants are enhanced by loss of $d y f-7$. Heads of (A) wild-type, (B) dyf-7(ns119), (C) dig-1(n1321), and (D) dig-1(n1321); dyf-7(ns119) animals at the L4 stage, showing dendrite extension defects. Arrowheads, dendrite endings. A severely shortened dendrite is boxed and magnified in (C). Animals express klp-6pro:GFP. (E) Quantification of phenotype expressivity. Dendrite lengths were measured as a fraction of the distance from the cell body to the nose tip. Gray bars, individual dendrites; black bars, population mean. $n>90$ per genotype except wild-type $n=30$. $p$-values, Mann-Whitney $\mathrm{U}$ test with Bonferroni correction.

\section{Discussion}

The functions of ECM are only beginning to be understood. Here, we describe additional phenotypes resulting from loss of DIG-1, a giant ECM protein, that shed light on its roles in maintaining the integrity of the nervous system. Although the developmental mechanisms that produce these phenotypes remain unclear, each of them can be interpreted in terms of cellular breakage: fragmentation of the amphid sheath; constriction and breakage of the BAG dendrite near its ending; and detachment of IL2 dendrites in a manner similar to the rupture of glial epithelial tubes caused by loss of DYF-7 [9].

Previous phenotypes associated with DIG-1 had pointed most strongly to roles in adhesion. These include the displaced gonad defects for which dig-1 is named [34]; defasciculation of amphid dendrite bundles and wandering of other dendrites including those of the IL2 neurons [10,11]; defects in neuronal cell body positioning [12,28]; and "flip-over" defects of several axons (for example: PVQ, AVK, and RMEV) in which axons are displaced across the midline and then return to their correct path [12]. Several of these phenotypes, including axon flip-over, arise progressively throughout larval development and are suppressed by inhibiting locomotion, suggesting they are caused by mechanical forces due to growth and movement [12]. One interpretation of these defects is that DIG-1 normally acts as an adhesion protein to keep neurites tightly attached to their correct partners.

Our results suggest a different, but not mutually exclusive, role in which DIG-1 creates a barrier between tightly packed structures in the nervous system. For example, DIG-1 may contribute structurally to the overall basal ECM that separates cells. Alternatively, it may form a coat around the extracellular surfaces of neurites, forming a barrier between cells even in the absence of an ultrastructurally visible basement membrane. In the context of fasciculation, this barrier might prevent neurites from contacting inappropriate partners to which they would promiscuously adhere. In support of this view, we note that in the case of IL2 defasciculation and axon flip-over, the neurites do not appear to lose adhesivity 
but instead they become tightly adhered to incorrect partners. In the context of preventing cellular breakage, this barrier would protect neurons and glia from neighboring structures that would otherwise impinge on them and cause fragmentation or rupture. In support of this view, we note that the BAG dendrite appears to break at a stereotyped position where it is normally thinner, as if under pressure from neighboring structures. It is less clear how this would cause fragmentation of the amphid sheath glial cell, but possibilities include aberrant adhesion with inappropriate neighbors or leakage through a perforated ECM that could cause shedding of small pieces of the glial cell as the animal grows and moves.

Consistent with the idea that DIG-1 contributes to basal ECM barriers between neurons, ultrastructural analysis has revealed defects in the basement membrane of dig-1 mutants [12]. By contrast, DYF-7 is a component of apical ECM that lines the lumen of developing epithelial tubes [9]. This suggests a surprising interplay between basal and apical ECM during IL2 dendrite extension. One speculative possibility is that while apical ECM acts in the lumen of developing epithelial (or glial) tubes to prevent rupture [40], basal $\mathrm{ECM}$ creates a protective barrier around the structure that reduces the overall mechanical stress it experiences during morphogenesis.

A perplexing feature of dig-1 phenotypes is their diversity and cell-type specificitysome ventral cord axons are affected more than others [12], BAG undergoes breakage while a similar neuron, URX, is unaffected (our unpublished observations), and IL2 neurons are affected during embryonic development while other cell types are only affected near adulthood [11,12]. One possibility is that these phenotypic differences reflect the local environment around each cell, including the forces exerted on it throughout development by neighboring structures. Overall, this model suggests that DIG-1 may play a role in preventing inappropriate cell-cell interactions that is as important to nervous system organization as promoting the correct contacts.

Supplementary Materials: The following are available online at https://www.mdpi.com/article/ $10.3390 / \mathrm{jdb} 9040042 / \mathrm{s} 1$, Figure S1: Isolation of mutants with altered glial or neuronal morphology, Table S1: Strains used in this study, Table S2: Transgenes used in this study, Table S3: Alleles generated in this study.

Author Contributions: Investigation, M.K.C., E.R.C. and K.M.; Supervision, M.G.H. All authors have read and agreed to the published version of the manuscript.

Funding: This research was funded by National Institutes of Health, grant number F31NS103371, R01NS112343 and R01GM108754.

Acknowledgments: We gratefully acknowledge WormBase; the CGC, which is funded by NIH Office of Research Infrastructure Programs (P40 OD010440); and funding from the NSF Graduation Research Fellowship Program (E.R.C.), National Institutes of Health (F31NS103371 to E.R.C.; R01GM108754 and R01NS112343 to M.G.H.), and the Hearst Fellows Program at Harvard University (K.M.).

Conflicts of Interest: The authors declare no conflict of interest.

\section{References}

1. Lamkin, E.R.; Heiman, M.G. Coordinated Morphogenesis of Neurons and Glia. Curr. Opin. Neurobiol. 2017, 47, 58-64. [CrossRef]

2. Josephson, M.P.; Miltner, A.M.; Lundquist, E.A. Nonautonomous Roles of MAB-5/Hox and the Secreted Basement Membrane Molecule SPON-1/F-Spondin in Caenorhabditis elegans Neuronal Migration. Genetics 2016, 203, 1747-1762. [CrossRef]

3. Lang, A.E.; Lundquist, E.A. The Collagens DPY-17 and SQT-3 Direct Anterior-Posterior Migration of the Q Neuroblasts in C. elegans. J. Dev. Biol. 2021, 9, 7. [CrossRef]

4. Wang, X.; Liu, J.; Zhu, Z.; Ou, G. The Heparan Sulfate-Modifying Enzyme Glucuronyl C5-Epimerase HSE-5 Controls Caenorhabditis elegans Q Neuroblast Polarization during Migration. Dev. Biol. 2015, 399, 306-314. [CrossRef] [PubMed]

5. Yang, Y.; Lee, W.S.; Tang, X.; Wadsworth, W.G. Extracellular Matrix Regulates UNC-6 (Netrin) Axon Guidance by Controlling the Direction of Intracellular UNC-40 (DCC) Outgrowth Activity. PLoS ONE 2014, 9, e97258. [CrossRef] [PubMed]

6. Blanchette, C.R.; Perrat, P.N.; Thackeray, A.; Bénard, C.Y. Glypican Is a Modulator of Netrin-Mediated Axon Guidance. PLoS Biol. 2015, 13, e1002183. [CrossRef] [PubMed] 
7. Celestrin, K.; Díaz-Balzac, C.A.; Tang, L.T.H.; Ackley, B.D.; Bülow, H.E. Four Specific Immunoglobulin Domains in UNC52/Perlecan Function with NID-1/Nidogen during Dendrite Morphogenesis in Caenorhabditis elegans. Development 2018, 145, dev158881. [CrossRef] [PubMed]

8. Liang, X.; Dong, X.; Moerman, D.G.; Shen, K.; Wang, X. Sarcomeres Pattern Proprioceptive Sensory Dendritic Endings through UNC-52/Perlecan in C. elegans. Dev. Cell 2015, 33, 388-400. [CrossRef]

9. Low, I.I.C.; Williams, C.R.; Chong, M.K.; McLachlan, I.G.; Wierbowski, B.M.; Kolotuev, I.; Heiman, M.G. Morphogenesis of Neurons and Glia within an Epithelium. Development 2019, 146. [CrossRef]

10. Yip, Z.C.; Heiman, M.G. Ordered Arrangement of Dendrites within a C. elegans Sensory Nerve Bundle. eLife 2018, 7, e35825. [CrossRef] [PubMed]

11. Burket, C.T.; Higgins, C.E.; Hull, L.C.; Berninsone, P.M.; Ryder, E.F. The C. elegans Gene Dig-1 Encodes a Giant Member of the Immunoglobulin Superfamily That Promotes Fasciculation of Neuronal Processes. Dev. Biol. 2006, 299, 193-205. [CrossRef] [PubMed]

12. Bénard, C.Y.; Boyanov, A.; Hall, D.H.; Hobert, O. DIG-1, a Novel Giant Protein, Non-Autonomously Mediates Maintenance of Nervous System Architecture. Development 2006, 133, 3329-3340. [CrossRef] [PubMed]

13. Bénard, C.Y.; Blanchette, C.; Recio, J.; Hobert, O. The Secreted Immunoglobulin Domain Proteins ZIG-5 and ZIG-8 Cooperate with L1CAM/SAX-7 to Maintain Nervous System Integrity. PLoS Genet. 2012, 8, e1002819. [CrossRef] [PubMed]

14. Blanchette, C.R.; Thackeray, A.; Perrat, P.N.; Hekimi, S.; Bénard, C.Y. Functional Requirements for Heparan Sulfate Biosynthesis in Morphogenesis and Nervous System Development in C. elegans. PLoS Genet. 2017, 13, e1006525. [CrossRef] [PubMed]

15. Desse, V.E.; Blanchette, C.R.; Nadour, M.; Perrat, P.; Rivollet, L.; Khandekar, A.; Bénard, C.Y. Neuronal Post-Developmentally Acting SAX-7S/L1CAM Can Function as Cleaved Fragments to Maintain Neuronal Architecture in C. elegans. Genetics 2021, 218 , iyab086. [CrossRef]

16. Cizeron, M.; Granger, L.; Bülow, H.E.; Bessereau, J.-L. Specific Heparan Sulfate Modifications Stabilize the Synaptic Organizer MADD-4/Punctin at C. elegans Neuromuscular Junctions. Genetics 2021, 218, iyab073. [CrossRef]

17. Pinan-Lucarré, B.; Tu, H.; Pierron, M.; Cruceyra, P.I.; Zhan, H.; Stigloher, C.; Richmond, J.E.; Bessereau, J.-L. C. elegans Punctin Specifies Cholinergic versus GABAergic Identity of Postsynaptic Domains. Nature 2014, 511, 466-470. [CrossRef]

18. Platsaki, S.; Zhou, X.; Pinan-Lucarré, B.; Delauzun, V.; Tu, H.; Mansuelle, P.; Fourquet, P.; Bourne, Y.; Bessereau, J.-L.; Marchot, P. The Ig-like Domain of Punctin/MADD-4 Is the Primary Determinant for Interaction with the Ectodomain of Neuroligin NLG-1. J. Biol. Chem. 2020, 295, 16267-16279. [CrossRef]

19. Zhou, X.; Vachon, C.; Cizeron, M.; Romatif, O.; Bülow, H.E.; Jospin, M.; Bessereau, J.-L. The HSPG Syndecan Is a Core Organizer of Cholinergic Synapses. J. Cell Biol. 2021, 220, e202011144. [CrossRef]

20. Lázaro-Peña, M.I.; Díaz-Balzac, C.A.; Bülow, H.E.; Emmons, S.W. Synaptogenesis Is Modulated by Heparan Sulfate in Caenorhabditis elegans. Genetics 2018, 209, 195-208. [CrossRef] [PubMed]

21. Fan, J.; Ji, T.; Wang, K.; Huang, J.; Wang, M.; Manning, L.; Dong, X.; Shi, Y.; Zhang, X.; Shao, Z.; et al. A Muscle-Epidermis-Glia Signaling Axis Sustains Synaptic Specificity during Allometric Growth in Caenorhabditis elegans. eLife 2020, 9, e55890. [CrossRef]

22. Heiman, M.G.; Shaham, S. DEX-1 and DYF-7 Establish Sensory Dendrite Length by Anchoring Dendritic Tips during Cell Migration. Cell 2009, 137, 344-355. [CrossRef] [PubMed]

23. Cebul, E.R.; McLachlan, I.G.; Heiman, M.G. Dendrites with Specialized Glial Attachments Develop by Retrograde Extension Using SAX-7 and GRDN-1. Development 2020, 147, dev180448. [CrossRef]

24. Cohn, J.A.; Cebul, E.R.; Valperga, G.; Brose, L.; de Bono, M.; Heiman, M.G.; Pierce, J.T. Long-Term Activity Drives Dendritic Branch Elaboration of a C. elegans Sensory Neuron. Dev. Biol. 2020, 461, 66-74. [CrossRef] [PubMed]

25. Fan, L.; Kovacevic, I.; Heiman, M.G.; Bao, Z. A Multicellular Rosette-Mediated Collective Dendrite Extension. eLife 2019, 8, e38065. [CrossRef] [PubMed]

26. Nechipurenko, I.V.; Olivier-Mason, A.; Kazatskaya, A.; Kennedy, J.; McLachlan, I.G.; Heiman, M.G.; Blacque, O.E.; Sengupta, P. A Conserved Role for Girdin in Basal Body Positioning and Ciliogenesis. Dev. Cell 2016, 38, 493-506. [CrossRef]

27. Ward, S.; Thomson, N.; White, J.G.; Brenner, S. Electron Microscopical Reconstruction of the Anterior Sensory Anatomy of the Nematode Caenorhabditis elegans. J. Comp. Neurol. 1975, 160, 313-337. [CrossRef]

28. Johnson, R.P.; Kramer, J.M. Neural Maintenance Roles for the Matrix Receptor Dystroglycan and the Nuclear Anchorage Complex in Caenorhabditis elegans. Genetics 2012, 190, 1365-1377. [CrossRef]

29. Zallen, J.A.; Kirch, S.A.; Bargmann, C.I. Genes Required for Axon Pathfinding and Extension in the C. elegans Nerve Ring. Development 1999, 126, 3679-3692. [CrossRef]

30. Doitsidou, M.; Poole, R.J.; Sarin, S.; Bigelow, H.; Hobert, O. C. Elegans Mutant Identification with a One-Step Whole-GenomeSequencing and SNP Mapping Strategy. PLoS ONE 2010, 5, e15435. [CrossRef]

31. Minevich, G.; Park, D.S.; Blankenberg, D.; Poole, R.J.; Hobert, O. CloudMap: A Cloud-Based Pipeline for Analysis of Mutant Genome Sequences. Genetics 2012, 192, 1249-1269. [CrossRef]

32. Chen, H.; Hughes, D.D.; Chan, T.A.; Sedat, J.W.; Agard, D.A. IVE (Image Visualization Environment): A Software Platform for All Three-Dimensional Microscopy Applications. J. Struct. Biol. 1996, 116, 56-60. [CrossRef]

33. Schindelin, J.; Arganda-Carreras, I.; Frise, E.; Kaynig, V.; Longair, M.; Pietzsch, T.; Preibisch, S.; Rueden, C.; Saalfeld, S.; Schmid, B.; et al. Fiji: An Open-Source Platform for Biological-Image Analysis. Nat. Methods 2012, 9, 676-682. [CrossRef] 
34. Thomas, J.H.; Stern, M.J.; Horvitz, H.R. Cell Interactions Coordinate the Development of the C. Elegans Egg-Laying System. Cell 1990, 62, 1041-1052. [CrossRef]

35. Doroquez, D.B.; Berciu, C.; Anderson, J.R.; Sengupta, P.; Nicastro, D. A High-Resolution Morphological and Ultrastructural Map of Anterior Sensory Cilia and Glia in Caenorhabditis elegans. eLife 2014, 3, e01948. [CrossRef] [PubMed]

36. Mizeracka, K.; Rogers, J.M.; Shaham, S.; Bulyk, M.L.; Heiman, M.G. Lineage-Specific Control of Convergent Differentiation by a Forkhead Repressor. Development 2021, 148, dev199493. [CrossRef] [PubMed]

37. Zhang, A.; Noma, K.; Yan, D. Regulation of Gliogenesis by Lin-32/Atoh1 in Caenorhabditis elegans. G3 (Bethesda) 2020, 10, 3271-3278. [CrossRef]

38. Zhang, A.; Guan, Z.; Ockerman, K.; Dong, P.; Guo, J.; Wang, Z.; Yan, D. Regulation of Glial Size by Eicosapentaenoic Acid through a Novel Golgi Apparatus Mechanism. PLoS Biol. 2020, 18, e3001051. [CrossRef]

39. Razzauti, A.; Laurent, P. Ectocytosis prevents accumulation of ciliary cargo in C. elegans sensory neurons. eLife 2021, 10, e67670. [CrossRef]

40. Sundaram, M.V.; Cohen, J.D. Time to Make the Doughnuts: Building and Shaping Seamless Tubes. Semin. Cell Dev. Biol. 2017, 67, 123-131. [CrossRef] 